\title{
Los híbridos de Pleopeltis (Polypodiaceae, Polypodiophyta) en México: novedades y precisiones
}

\section{The Pleopeltis (Polypodiaceae, Polypodiophyta) hybrid in Mexico: novelties and precisions}

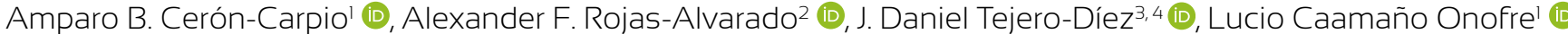

1 Benemérita Universidad Autónoma de Puebla, Herbario y Jardín Botánico, Vicerrectoría de Investigación y Estudios de Posgrado; Ciudad Universitaria, Avenida San Claudio s.n., Colonia San Manuel, 72570 Puebla, Puebla, México.

2 Universidad Nacional de Costa Rica, Escuela de Ciencias Biológicas, Apdo. postal 86-3000, Heredia, Costa Rica.

3 Universidad Nacional Autónoma de México, Facultad de Estudios Superiores Iztacala, Carrera de Biología, Avenida de Los Barrios 1, Los Reyes Iztacala, Tlalnepantla 54090 Estado de México, México.

4 Autor para la correspondencia: tejero@unam.mx

Citar como:

Cerón-Carpio, A. B., A. F. Rojas-Alvarado, J. D. Tejero-Díez y L. C. Onofre. 2017. Los híbridos de Pleopeltis (Polypodiaceae, Polypodiophyta) en México: novedades y precisiones. Acta Botanica Mexicana 119: 101-114. DOI: http://dx.doi.org/10.21829/ abm119.2017.1234

Recibido: 20 de marzo de 2015. Revisado: 23 de julio de 2015. Aceptado: 16 de noviembre de 2016.

DOI:

http://dx.doi.org/10.21829/abm119.2017.1234

\section{Resumen:}

Antecedentes y Objetivos: En México, hasta el momento, se conocen cuatro híbridos de Pleopeltis. Estos híbridos naturales forman parte de los procesos de especiación y contribuyen a aumentar la diversidad taxonómica del género en el país. En este ensayo se dan a conocer dos nuevos híbridos recolectados en la Sierra Madre Oriental de Puebla, México y se reconoce formalmente otro híbrido. Métodos: Estos híbridos nuevos se definieron mediante el estudio comparativo de caracteres morfológicos en la arquitectura laminar y tipo de indumento.

Resultados clave: Se reconocen como híbridos nuevos a Pleopeltis $\times$ gracilis y Pleopeltis $\times$ pueblensis y se publica de forma válida a Pleopeltis $\times$ arreguiniae.

Conclusiones: Con base en el reconocimiento de estos nuevos nothotaxa, México cuenta con siete híbridos en Pleopeltis. Aparte de presentar tablas comparativas, se ofrece una clave para la correcta determinación de estos nothotaxa.

Palabras clave: clave dicotómica, helechos, Hidalgo, Puebla, Pteridofita.

\section{ABSTRACT:}

Background and Aims: In Mexico four hybrids in Pleopeltis are known so far. The presence of these natural hybrids of Polypodiaceae is part of the speciation processes and contributes to the higher taxonomic diversity in this country. In this paper we disclose two new hybrids collected in the Sierra Madre Oriental in Puebla, Mexico, whereas another hybrid is formally recognized.

Methods: These new hybrids are defined by a comparative study of morphological characters in laminar architecture and indument type.

Key results: We describe two new hybrids: Pleopeltis $\times$ gracilis and $P$. $\times$ pueblensis and validly publish $P$. $\times$ arreguiniae.

Conclusions: With these nothotaxa, Mexico has now seven hybrids of Pleopeltis. Additionally, to support correct identification, we present comparative tables and a key.

Key words: dichotomous key, ferns, Pteridophytes, state of Hidalgo, state of Puebla.

\section{INTRODUCCIÓN}

La presencia de híbridos en Pleopeltis Humb. \& Bonpl. ex Willd. (Polypodiaceae) ha sido documentada en varias ocasiones (Gómez-Pignataro, 1975; Wagner y Wagner, 1975; Anthony y Schelpe, 1985; Mickel y Beitel, 1987; Arreguín-Sánchez et al., 2004; Farfán-Roldán et al., 2006), de tal forma que Smith y Tejero-Díez (2014) reconocen para el género un total de ocho nothotaxa, cuatro de las cuales son endémicas a México, tres ocurren en Sudamérica y una en Sudáfrica. Inicialmente varios de estos híbridos se consideraron como intergenéricos entre Polypodium L. y Pleopeltis $(\times$ Pleopodium Mickel $\&$ Beitel), un aspecto considerado contradic- 
torio, por lo cual Smith y Tejero-Díez (2014) reformaron el concepto de Pleopeltis. De acuerdo con la redefinición mencionada, actualmente se considera a Pleopeltis como un género de 90 especies y ocho nothoespecies y se diferencia del resto de los géneros de Polypodiaceae en América principalmente por tener escamas peltadas en la lámina (Otto et al., 2009; Smith y Tejero-Díez, 2014). La mayoría de los híbridos descritos en Pleopeltis (Mickel y Beitel, 1987) parecen originarse de la cruza entre especies del grupo P. macrocarpa (Bory ex Willd.) Kaulf. de hoja simple (sensu Smith y Tejero-Díez, 2014): Pleopeltis astrolepis (Liebm.) E. Fourn., P. complanata (Weath.) E.A. Hooper, P. conzattii (Weath.) R.M. Tryon \& A.F. Tryon, P. crassinervata (Fée) T. Moore, P. fructuosa (Maxon \& Weath.) Lellinger, P. intermedia M. Kessler \& A.R. Sm., P. mexicana (Fée) Mickel \& Beitel, P. polylepis (A. Roem. ex Kunze) T. Moore y P. stolzei A.R. Sm.) con especies de hojas pinnatífidas o pinnadas, como P. fallax (Schltdl. \& Cham.) Mickel \& Beitel, P. thyssanolepis (A. Braun ex Klotzsch) E.G. Andrews \& Windham y P. polypodioides (L.) E.G. Andrews y Windham. Dado que producen hojas anómalas, estos híbridos son faciles de diferenciar. En contraste, los híbridos intragrupales son superficialmente similares a las especies paternas y, por lo tanto, son difíciles de distinguir y no hay reportes de su presencia. Hasta antes del presente estudio, Mickel y Beitel (1987), Mickel y Smith (2004), así como Smith y Tejero-Díez (2014), reconocían para México los siguientes cuatro híbridos.

1) Pleopeltis $\times$ bartlettii (Weath.) A.R. Sm. y Tejero = Polypodium bartlettii Weath. (1935: 56) = $\times$ Pleopodium bartlettii (Weath.) Mickel \& Beitel (1987: 20, t. 1K-L). Reconocido como posible híbrido entre Pleopeltis polylepis (A. Roem. ex Kunze) T. Moore y P. polypodioides (L.) E.G. Andrews y Windham, registrado para Tamaulipas.

2) Pleopeltis $\times$ melanoneuron Mickel \& Beitel (1987: 25, f. 2K). Reconocido como posible híbrido entre Pleopeltis crassinervata (Fée) T. Moore \& P. fallax (Schltdl. y Cham.) Mickel y Beitel, registrado para Veracruz y recientemente recolectado en Pue- bla (Cuetzalán; E. Schuettpelz et al. 1787, 1796 y 1797; F!, MEXU!).

3) Pleopeltis $\times$ sordidula (Maxon y Weath.) Mickel \& Beitel (1987: 21). Reconocido como posible híbrido entre P. astrolepis (Liebm.) E. Fourn. y P. fallax (Schltdl. \& Cham.) Mickel \& Beitel, registrado para Veracruz (Córdoba) y recientemente recolectado en Tlaxcala (Arreguín-Sánchez et al., 2004).

4) Pleopeltis $\times$ tricholepis (Mickel \& Beitel) A.R. Sm. y Tejero $=\times$ Pleopodium tricholepis Mickel and Beitel (1987: 17). Reconocido como posible híbrido entre Pleopeltis mexicana (Fée) Mickel \& Beitel y Pleopeltis thyssanolepis (A. Braun ex Klotzsch) E.G. Andrews y Windham, registrado para Oaxaca.

Debido a que el hallazgo de estas entidades taxonómicas es casual y sus poblaciones casi siempre constan de una o pocas colonias, generalmente las diagnosis adolecen de un buen muestreo biométrico. Ello puede ocasionar confusiones en los límites morfológicos de cada taxon híbrido al desconocerse qué caracteres son dominantes y cuáles obedecen a una clina de variación entre los caracteres extremos heredados de los padres. Otro problema en la definición de los híbridos en este género (y en general en los helechos) es que no existe un estándar sobre la proporción de esporas viables/inviables, que permitan indicar cuándo se puede considerar una especie o simplemente un híbrido (Wagner et al., 1986). Esto ha llevado a cambios de rango taxonómico, por ejemplo: $\times$ Pleopodium fallacissimum (Maxon) Mickel \& Beitel a Pleopeltis fallacissima (Maxon) A.R. Sm. y Tejero (Mickel y Smith, 2004), cuando se hallan mayor cantidad de poblaciones. Un caso contrario es cuando se revisan las esporas y se modifica el estado de rango tal como en Polypodium bartlettii que pasó a Pleopeltis $\times$ bartlettii.

En los trabajos pteridoflorísticos que los autores llevaron a cabo en la Sierra Norte del estado de Puebla, (municipios de Ahuazotepec y Xicotepec de Juárez), se localizaron colonias de Pleopeltis con hojas anómalas por el tipo de división que presentan. La presencia de híbridos en la naturaleza no solo es de importancia florística y 
ecológica, sino que permite documentar procesos de especiación que, particularmente en los helechos, destacan por tener patrones reticulados (Wagner, 1969; Barrington et al., 1989; Haufler et al., 2000). Además, el reconocimiento taxonómico de los híbridos en Pleopeltis podrá permitir en un futuro esclarecer las relaciones taxonómicas infragenéricas que actualmente se encuentran en forma de hipótesis (Smith y Tejero-Díez, 2014), así como probables rutas de su evolución reticular tal como ha sucedido con las especies del grupo de Polypodium vulgare L. (Haufler et al., 1995; Sigel et al., 2014).

Por lo anterior, en este trabajo se hace una revisión de los caracteres morfológicos de híbridos de Pleopeltis descritos previamente, con la finalidad de tener una mejor comprensión de la circunscripción taxonómica de los nothotaxa recolectados y proponer así dos nuevos híbridos y el reconocimiento formal de otro. Además, se propone una clave dicotómica para formalizar los caracteres diagnósticos y facilitar la determinación de híbridos del género Pleopeltis en México.

\section{Materiales y Métodos}

En el contexto de un muestreo florístico, se recolectaron especímenes de Pleopeltis en los municipios de Ahuazotepec y Xicotepec de Juárez en la Sierra Norte del estado de Puebla, el primero con hojas anómalas y el segundo con caracteres intermedios. En el caso de los ejemplares recolectados en el municipio de Ahuazotepec, se definió su carácter híbrido mediante el análisis simple de las esporas al microscopio óptico (Nikon Eclipse E200, Tokio, Japón) preparados con la técnica de Plá’ Dalmau (Aguilar-Rodríguez, 1998); la malformación (anómalas sin forma típica monolete, oblongo-arriñonada) de $82 \%$ de las esporas contabilizadas, junto con la irregularidad de la arquitectura de la lámina, indicaron su origen híbrido. En el caso del espécimen recolectado en Xicotepec, carente de soros, los caracteres intermedios de la lámina y venación (observada mediante la aplicación de la técnica de diafanizado (Aguilar-Rodríguez, 1998) permitieron suponer su carácter híbrido. Los ejemplares se intentaron determinar por medio de los trabajos de Mickel y Beitel (1987) y
Mickel y Smith (2004), lo que fue infructuoso. Además de considerar los estudios mencionados, se observaron ejemplares tipo de herbario incorporados en JSTOR Global Plants (2016).

La revisión de la literatura pertinente llevó a considerar otra entidad híbrida de reciente cuño (Farfán-Roldán et al., 2006), pero cuya publicación no fue válida según el ICBN (McNeill et al., 2012), situación que se rectifica en este estudio.

\section{Resultados}

\section{Taxonomía}

A continuación se describen los nuevos nothotaxa.

Pleopeltis $\times$ gracilis A. Rojas \& Tejero, hybr. nov. Fig. 1.

TIPO: MÉXICO. Puebla, municipio de Xicotepec de Juárez, 20¹6'32"N, 9758'02"O, 1170 m, 1.V.2015, $A$. Rojas y D. Tejero-Díez 11060 (holotipo: MEXU!, isotipos: CR!, HUAP!).

Hybrid between Pleopeltis fallax (Schltdl. \& Cham.) Mickel \& Beitel and P. villagranii (Copel.) A.R. Sm. \& Tejero, with intermediate characters. Rhizomes slim, long creeping, green; rhizome scales ovate-apiculate (to long caudate), not comose (with exceptions), bicolorous; leaves distant, with stipes (and rachis) green, blade lanceolate to linear-lanceolate, pinnate, gradually tapering towards the apex, pinnae with (1-)2-3 pairs of lobo-crenate segments; indument scales scattered on both surfaces, adaxially more sparsely, orbicular to ovate-apiculate or long caudate, center obscure brown to black, margin pale brown, fimbriate at base, margins dentate; sori not known.

Rizoma largamente rastrero, 0.4-0.8 mm de diámetro; escamas del rizoma dimorfas, las del rizoma maduro 0.4-0.6 de largo, estrechamente ovadas, acuminadas, bicoloras, el punto de adhesión constituido de celdillas ocluidas negras, margen ancho, membranoso, pardoamarillento, erosas en la base, márgenes con pequeños 

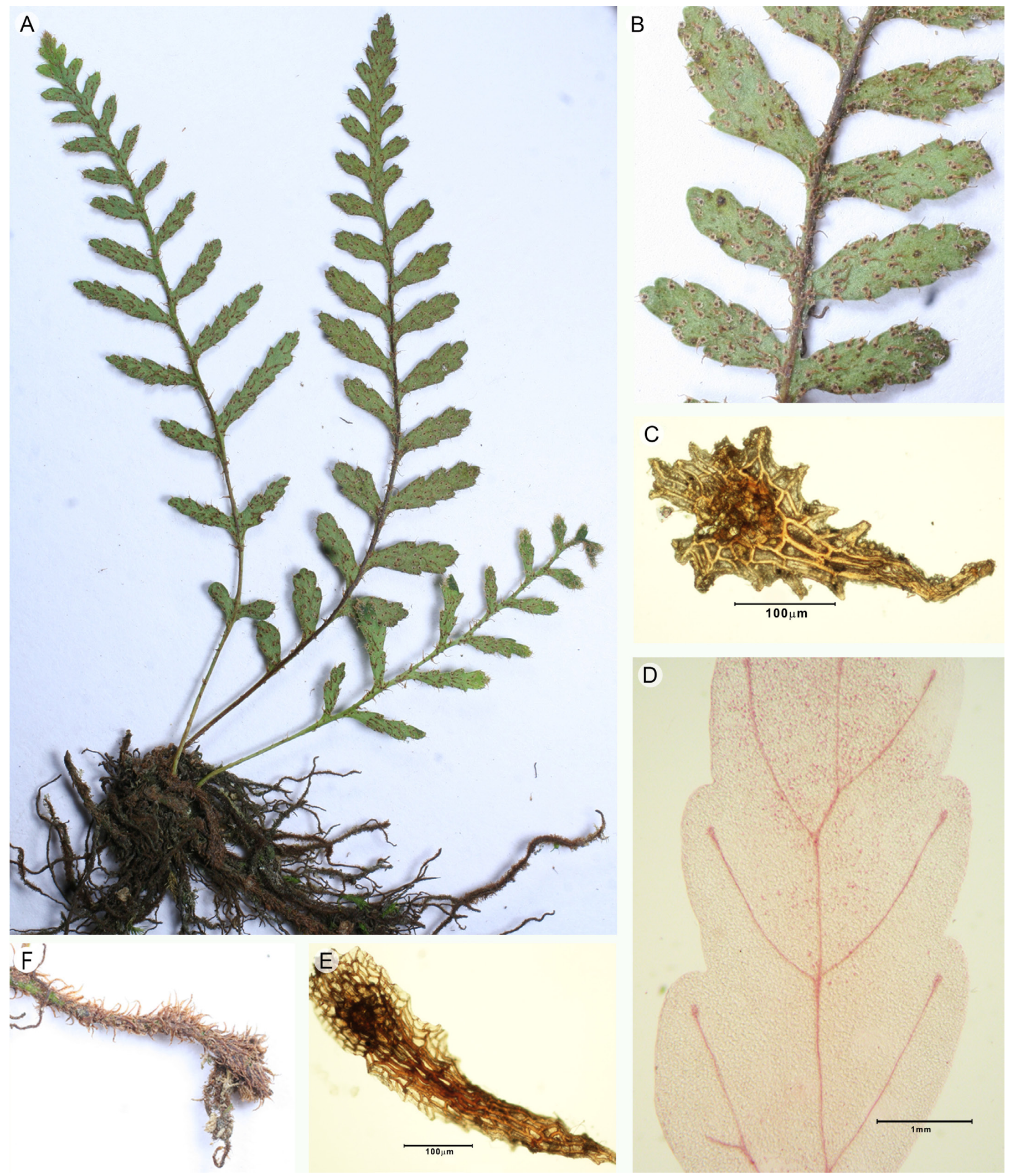

Figura 1: Detalles morfológicos de Pleopeltis × gracilis A. Rojas \& Tejero del holotipo (A. Rojas y D. Tejero-Díez 11060 MEXU). A. planta; B. anverso de la lámina; C. escama del anverso de la lámina; D. lámina diafanizada mostrando venación simple; E. rizoma; F. escama del rizoma. 
dientes, las del ápice del rizoma y base de las hojas 0.5-0.7 mm de largo, estrechamente lanceoladas, ápice atenuado, arqueadas a semi-erectas, bicoloras, con una franja central parda a pardo-oscuro de celdillas esclerosadas y semiocluidas, margen ancho, pardo-amarillento, inconspicuamente dentado, no comosas; hojas $2-8 \mathrm{~cm}$ de largo, distantes entre sí, (2-)7-15 mm; peciolo estrechamente alado, de 1/6-1/4 del largo de la hoja, 5-13 cm de largo, terete a cuadrangular, verdoso; lámina de contorno angostamente lanceolada a linear-lanceolada, de 1-6 $\times$ 0.4-1.5 cm, pinnada (o pinnatisectas, con segmentos unidos por las bases largamente decurrentes que forman un ala sobre el raquis $0.1-0.2 \mathrm{~mm}$ de ancho), gradualmente reducida hacia el ápice, pinnas estrechamente obovadas a lanceoladas, (1-)2-7 × (0.7-)1-2 mm, (3-)6-13 pares, distantes y alternadas o continuas y opuestas, sésiles con bases decurrentes, margen con (1-)2-5 pares de lobos o crenas, lobo apical agudo a redondeado, vena pajiza a parda (al menos basalmente), lustrosa, poco evidente hacia el ápice de la lámina, las secundarias no visibles; indumento de escamas esparcidas en ambas superficies (más densas en el envés), orbiculares a ovadas, con un ápice caudado a cirroso, 0.3-0.7 × 0.2-0.3 mm, con el centro pardo obscuro a negro y el margen hialino a pardo pálido, con el margen corto laciniado en el cuerpo y dentado en la cauda; soro no conocido pero un conjunto de escamas sorales o paráfisis presentes, ovado-peltadas, similar a las laminares.

Distribución y hábitat: Pleopeltis $\times$ gracilis hasta ahora solo se conoce para el municipio de Xicotepec de Juárez, en la Sierra Norte del estado de Puebla. Se encontró en una cerca viva de boj (Buxus sempervirens L.) que limita al hotel Xicotepec Plaza. Pleopeltis fallax y $P$. villagranii (Copel.) A.R. Sm. \& Tejero forman aquí colonias densas y conviven con Campyloneuron angustifolium (Sw.) Fée, Niphidium crassifolium (L.) Lellinger, Phlebodium pseudoaureum (Cav.) Lellinger, Pleopeltis cassinervata (Fée) T. Moore, P. furfuracea (Schltdl. \& Cham.) A.R. Sm. \& Tejero y P. plebeia (Schltdl. \& Cham.) A.R. Sm. \& Tejero.
Etimología: El epíteto específico hace referencia a sus hojas frágiles y angostas, con rizomas y peciolos muy delgados.

Comentarios: El espécimen descrito presenta caracteres intermedios que permiten dilucidar que uno de los progenitores es Pleopeltis fallax, el cual está caracterizado por presentar rizoma filamentoso con hojas pequeñas, lámina pinnada y pinnas pinatisectas, con las escamas laminares dispersas. Por las hojas lineares y el tipo de indumento en rizoma y hoja del híbrido se le puede emparentar con P. villagranii (Copel.) A.R. Sm. y Tejero (Cuadro 1). Este sería el primer híbrido entre Pleopeltis fallax y una especie del grupo de Pleopeltis furfuracea (Schltdl. \& Cham.) A.R. Sm. (ambas de lámina dividida) en México. Sin embargo, en Costa Rica ya se conoce Pleopeltis $\times$ pinnatisecta (Brade) A.R. Sm., un híbrido entre P. furfuracea y P. friedrichsthaliana (Kunze) A.R. Sm. y Tejero (Smith y Tejero-Díez, 2014).

Pleopeltis $\times$ gracilis se distingue fácilmente de sus conhíbridos mexicanos por tener una lámina de contorno linear-lanceolado y lámina pinnada con los segmentos estrechamente obovados, simétricamente lobadas.

Pleopeltis $\times$ pueblensis A.B. Cerón \& Tejero, hybr. nov. Fig. 2.

TIPO: MÉXICO. Puebla, municipio de Ahuazotepec, $1 \mathrm{~km}$ al norte de la cabecera municipal, cerca del cementerio, $20^{\circ} 3^{\prime} 6.3^{\prime \prime} \mathrm{N}, 9^{\circ} 9^{\prime} 53^{\prime \prime} \mathrm{O}, 2303 \mathrm{~m}$ s.n.m., 12.IX.2013, A. Cerón-Carpio y L. Caamaño Onofre 1547 (holotipo: HUAP!, isotipos: ENCB!, MEXU!).

Putative hybrid between Pleopeltis mexicana (Fée) Mickel \& Beitel and P. madrensis (J. Sm.) A.R. Sm. \& Tejero, with the following diagnostic characters. Rhizomes long creeping, covered with membranous ovate-apiculate scales; rhizome scales comose in distinct grades on dorsal surface, bicolorous with a dark central streak of sclerotic cells and pale margins, which 
Cuadro 1: Comparación de Pleopeltis $\times$ gracilis A. Rojas \& Tejero con sus posibles progenitores.

\begin{tabular}{|c|c|c|c|}
\hline Características & $\begin{array}{l}\text { P. fallax (Schltdl. \& Cham.) } \\
\text { Mickel \& Beitel }\end{array}$ & $\begin{array}{c}\text { P. } \times \text { gracilis } \\
\text { A. Rojas \& Tejero }\end{array}$ & $\begin{array}{c}\text { P. villagranii (Copel.) } \\
\text { A.R. Sm. \& Tejero }\end{array}$ \\
\hline Diámetro del rizoma & $0.5-0.8 \mathrm{~mm}$ & $0.5-0.8 \mathrm{~mm}$ & $1.5-2 \mathrm{~mm}$ \\
\hline Distancia entre hojas en el rizoma & $5-20 \mathrm{~mm}$ & $2-15 \mathrm{~mm}$ & $3-8(-15) \mathrm{mm}$ \\
\hline Comas en las escamas del rizoma & sí & algunas e inconspicuas & no \\
\hline Forma de las escamas del rizoma & $\begin{array}{l}\text { monomorfas; ovadas, adpresas, } \\
\text { diminutas }\end{array}$ & $\begin{array}{l}\text { dimorfas; ovadas, adpresas con } \\
\text { ápice acuminado y lanceoladas, } \\
\text { algo erectas o curvas con ápice } \\
\text { atenuado }\end{array}$ & $\begin{array}{l}\text { monomorfas: lanceoladas, } \\
\text { patentes o levemente adpresas }\end{array}$ \\
\hline Posición de las hojas & rígidas, erectas o hacia los lados & arqueadas & péndulas \\
\hline Longitud del peciolo & ca. $1 / 3$ del largo de la hoja & 1/4-1/6 del largo de la hoja & 1/8-1/4 del largo de la hoja \\
\hline Forma de la lámina & oblonga a angostamente deltada & $\begin{array}{l}\text { angostamente lanceolada a linear- } \\
\text { lanceolada }\end{array}$ & linear-oblonga \\
\hline & & & \\
\hline Forma de las escamas de la lámina & $\begin{array}{l}\text { lanceoladas, con ápice acuminado, } \\
\text { margen serrulado }\end{array}$ & $\begin{array}{l}\text { ovados caudadas a cirroso, } \\
\text { margen dentado a corto ciliado }\end{array}$ & $\begin{array}{l}\text { linear-lanceoladas a aciculares, } \\
\text { con ápice largamente atenuado, } \\
\text { margen eroso a fimbriado }\end{array}$ \\
\hline Paráfisis & ovado-lanceoladas & $\begin{array}{l}\text { desconocidas (solo material } \\
\text { vegetativo) }\end{array}$ & $\begin{array}{l}\text { lanceoladas, más grandes que las } \\
\text { laminares }\end{array}$ \\
\hline
\end{tabular}

are denticulate to erose; blade lanceolate to oblanceolate, basally pinnatisect and lobed to pinnatifid dista1ly, with proximal lobe free; laminar scales scattered on abaxial surface and glabrescent on adaxial surface, with round to ovate scales with apiculate or caudate apices, center obscure brown, margins pale brown, fimbriate to ciliate; midribs and secondary veins not visible; sori oblong, with orbicular, peltate scales similar to those in the lamina; spores bilateral, abortive, prominently verrucate. 

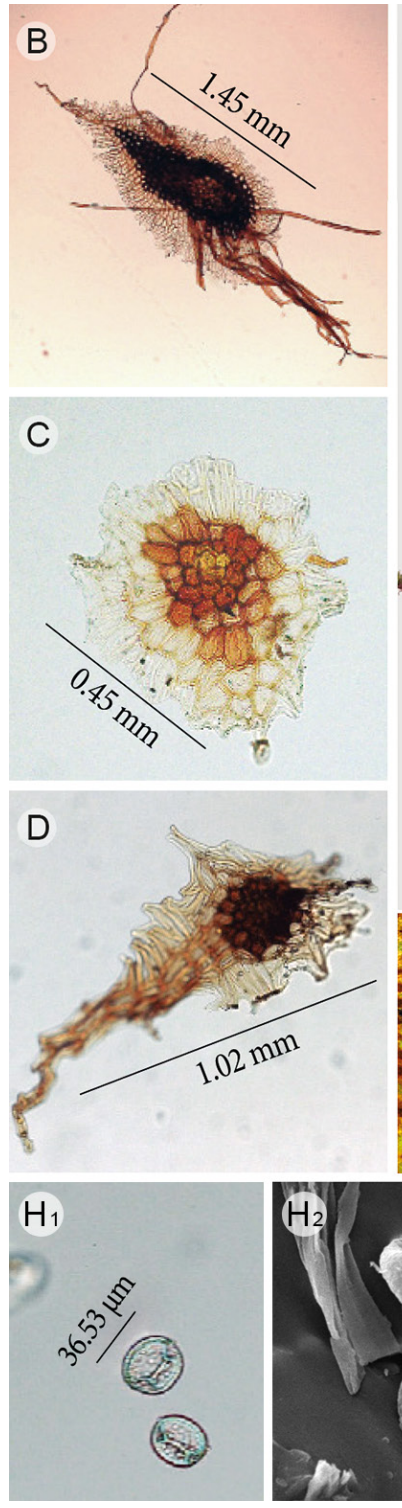
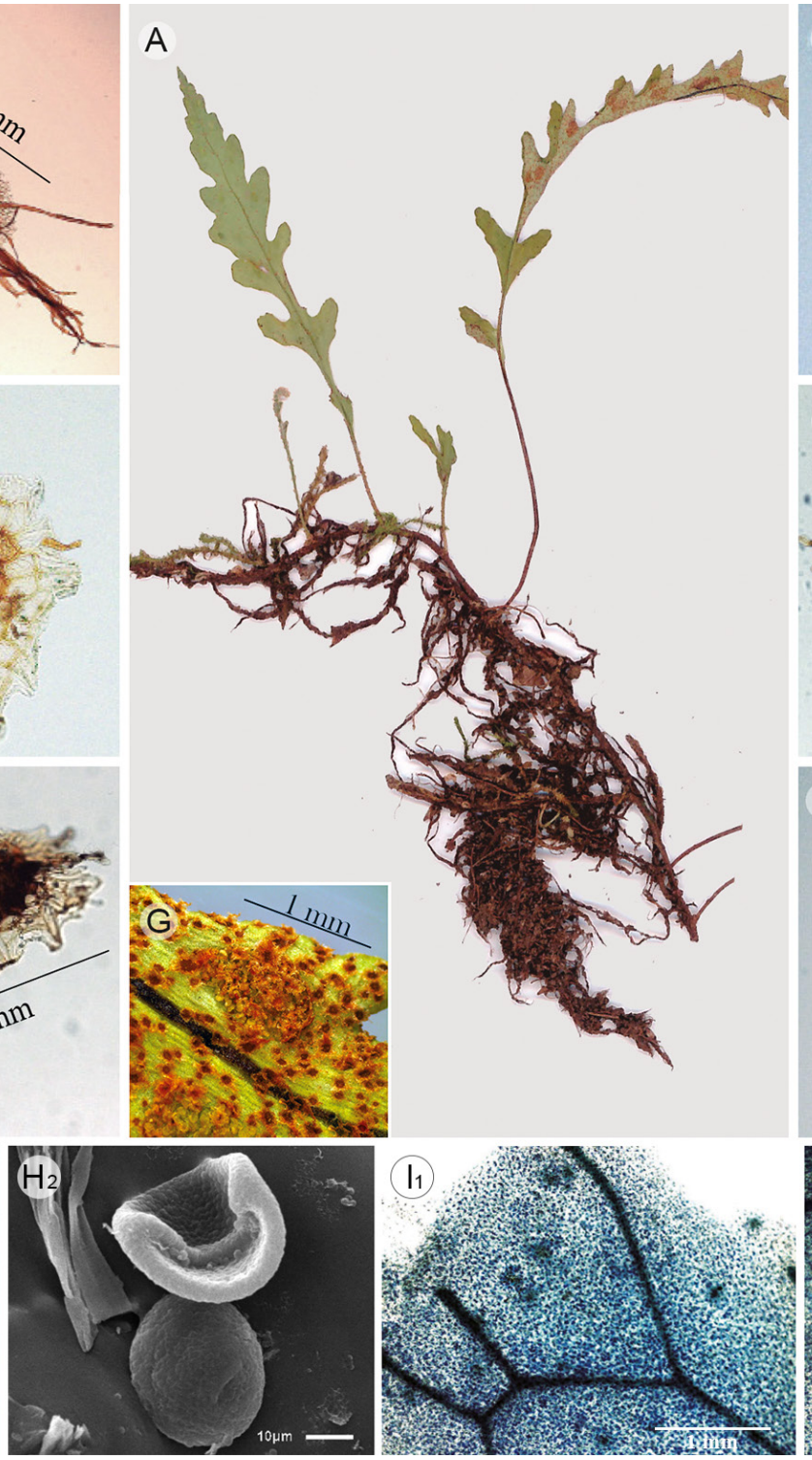
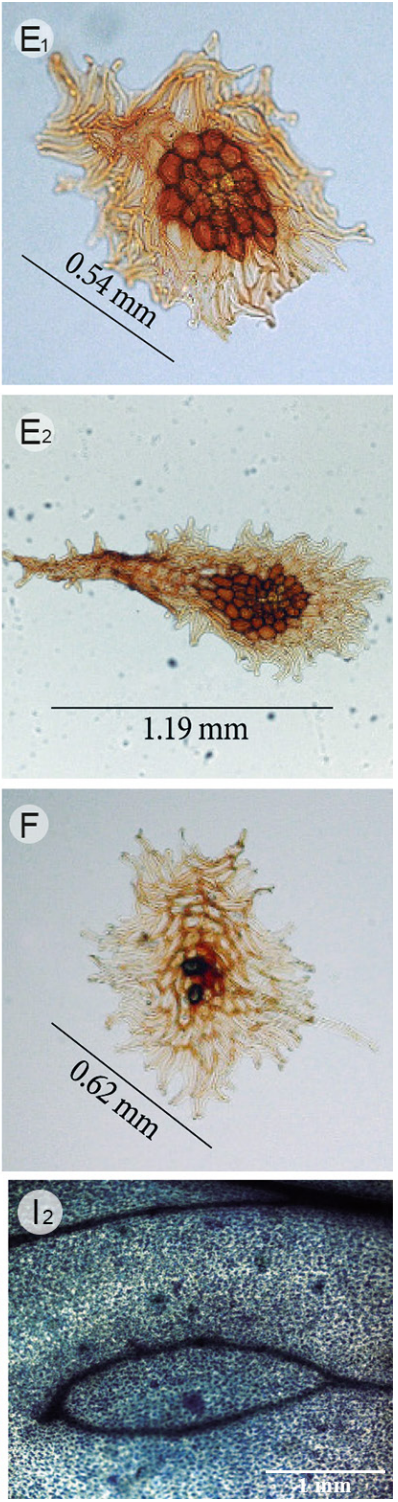

Figura 2: Detalles anatómicos de Pleopeltis × pueblensis del holotipo (A. Cerón-Carpio y L. Caamaño Onofre 1547, HUAP). A. planta herborizada; B. escama del rizoma; C. escama del peciolo; D. escama cara adaxial de la lámina; $E_{1}$. y $E_{2}$. escamas de la cara abaxial de la lámina; F. parafiso; G. lámina, cara abaxial. $\mathrm{H}_{1}$. esporas abortadas (microscopio óptico); $\mathrm{H}_{2}$. esporas abortivas (microscopio electrónico de barrido); $\mathrm{I}_{1}$ y $\mathrm{I}_{2}$. venación, 1 en la porción marginal, 2 en el cenosoro.

Rizoma largamente rastrero, 0.7-1 mm de diámetro; escamas del rizoma monomórficas, $0.8-1.5 \mathrm{~mm}$ de largo, ovado-apiculadas, bicoloras, al centro con una banda negra de celdillas subclatradas y margen ancho, membranoso, café pálido, con pequeños dientes a erosas, en el dorso del punto de inserción comosas en distintos grados de densidad y longitud; hojas 9-13 cm de largo, distantes a aproximadas; peciolo 1/4-1/5 del largo de la hoja, 2.5-4 cm de largo, terete, marrón; lámina lanceolada a oblanceolada, decurrente sobre el peciolo con las proyecciones alares estrechas que se desvanecen cerca de la base, 5-9.5 $\times 1.3-1.8 \mathrm{~cm}$, lobado-pinnatífida hacia el ápice a pinnatisecta hacia la base con los lóbulos proximales distantes, vena media obscura, poco evidente hacia el ápice de la lámina, las secundarias no visibles; indumento de escamas esparcidas en el envés y glabrescente en el haz, escamas 
del envés mixtas, de orbiculares a ovado-apiculadas y caudadas, 0.4-1 mm de diámetro, con el centro café obscuro y margen café claro, fimbriado a ciliado; soro oblongo, con escamas sorales (paráfisis) peltados, orbiculares a ovadolanceoladas, bicoloras, con centro obscuro y el margen café claro membranoso, margen fimbriado ciliado, 0.2$0.5 \mathrm{~mm}$ de diámetro; esporas monoletes, deformes, con verrugas en la superficie, 18-36 $\mu \mathrm{m}$ del diámetro mayor, 28-29 $\mu \mathrm{m}$ del menor en vista polar proximal.

Distribución y hábitat: Pleopeltis $\times$ pueblensis hasta ahora solo se conoce para el municipio de Ahuazotepec, en la llamada Sierra Norte del estado de Puebla. Se encontró en bosque de Pinus, con evidencias de perturbación.

Etimología: El epíteto específico hace referencia a la actual distribución conocida en el estado de Puebla.

Comentarios: De los híbridos en Pleopeltis hasta ahora descritos para México (Mickel y Smith 2004; Smith y Tejero-Díez, 2014), Pleopeltis $\times$ pueblensis es el único que presenta la lámina irregularmente pinnatisecta (a pinnada) en la base. Como varios de los híbridos encontrados en este género, las especies parentales son inciertas (Mickel y Beitel, 1987; Mickel y Smith, 2004) y hasta no probar con técnicas sofisticadas (una revisión de técnicas puede leerse en Barrington et al., 1989 y Chang et al., 2009), será imposible establecerlo con seguridad. Se podría especular que, por la lámina de contorno lanceolado, escamas del rizoma comosas y las de la lámina esparcida (sobre todo adaxialmente), con elementos dominantes orbiculares, uno de los progenitores podría ser Pleopeltis mexicana. La otra especie progenitora tendría que buscarse entre las especies de Pleopeltis pinatífidas, con escamas del rizoma de doble textura-color, sin escamas peciolares y muy pocas en la superficie adaxial de la lámina (estas escamas grandes y ovado-caudadas); tal vez alguna especie del grupo de Pleopeltis plebeia (Schltdl. \& Cham.) A.R. Sm. \& Tejero (Smith y Tejero-Díez, 2014). En el área donde se encontró $P$. × pueblensis, se recolectó de este grupo a Pleopeltis madrensis (J. Sm.) A.R. Sm. \&
Tejero, que cumple con algunos de los requisitos señalados y, además, al igual que el híbrido encontrado, sus esporas presentan grandes verrugas en la superficie (Cuadro 2). Otra especie de Pleopeltis recolectada en la localidad es P. polylepis var. polylepis; sin embargo, presenta la superficie de la hoja (peciolo y porción abaxial de la lámina) densamente escamosa. Este sería el primer híbrido entre una especie de hoja simple y otra del grupo de P. plebeia (sensu Smith y Tejero-Díez, 2014).

Pleopeltis $\times$ pueblensis se distingue fácilmente de sus conhíbridos mexicanos por tener una lámina de contorno lanceolado con los segmentos basales pinnatífido a pinnados (por lo menos con un lobo libre).

Finalmente, durante la revisión de la literatura pertinente para documentar este trabajo, se encontró que otras dos entidades híbridas de Pleopeltis habían sido propuestas, pero cuyos formatos de publicación no siguieron las reglas del ICBN (McNeill et al., 2012) de publicación efectiva y/o válida. Una de ellas, mencionada por ReyesCarrada (2012), no se expone en este trabajo por tratarse de una tesis que necesita ser publicada. El siguiente, donde Farfán-Roldán et al. (2006) propusieron la fórmula híbrida Pleopeltis polylepis var. polylepis $\times$ Polypodium guttatum Maxon (= Pleopeltis guttata (Maxon) E. G. Andrews y Windham, la cual no fue válidamente publicada de acuerdo a los artículos 32.4, H10.2 del ICBN (McNeill et al., 2012), por lo que necesita tener un holotipo y un epíteto correctamente dispuesto, acorde además a la nueva circunscripción de Pleopeltis (Smith y Tejero-Díez, 2014), por lo que a continuación se propone:

Pleopeltis $\times$ arreguiniae I. Farfán, Gómez-Alanís ex Tejero. hybr. nov. (fig. 10-18 in: Farfán-Roldán et al., 2006).

TIPO: MÉXICO. Hidalgo, municipio de Mineral del Chico, Parque Nacional El Chico, en bosque de pino y encino, $20^{\circ} 11^{\prime} 07^{\prime \prime} \mathrm{N}$ y $98^{\circ} 45^{\prime} 02^{\prime \prime} \mathrm{O}, 2880 \mathrm{~m}$ s.n.m., 25.IX.2005, I. Farfán-Roldán et al. s.n. (holotipo: ENCB). 
Cuadro 2: Comparación de Pleopeltis × pueblensis A.B. Cerón \& Tejero con sus progenitores putativos.

\begin{tabular}{|c|c|c|c|}
\hline Características & $\begin{array}{c}\text { P. mexicana } \\
\text { (Fée) Mickel \& Beitel }\end{array}$ & $\begin{array}{c}\text { P. × pueblensis } \\
\text { A.B. Cerón \& Tejero }\end{array}$ & $\begin{array}{c}\text { P. madrensis } \\
\text { (J. Sm.) A.R. Sm. \& Tejero }\end{array}$ \\
\hline Escamas del rizoma & $\begin{array}{l}\text { dimórficas; ovado-lanceoladas, } \\
\text { bicoloras y angostamente } \\
\text { lanceoladas, concoloras; } \\
\text { densamente comosas }\end{array}$ & $\begin{array}{l}\text { monomórficas, ovado-lanceoladas, } \\
\text { bicoloras, subclatradas con } \\
\text { margen membranoso pálido; } \\
\text { glabras a comosas }\end{array}$ & $\begin{array}{l}\text { monomórficas, ovado-lanceoladas, } \\
\text { bicoloras; clatradas con margen } \\
\text { membranoso pálido; no comosas }\end{array}$ \\
\hline Lámina & simple, entera & $\begin{array}{l}\text { lobado pinnatífida hacia el ápice, } \\
\text { pinnatisecta hacia la base, con } \\
\text { lobo(s) libre(s) }\end{array}$ & pinnatisecta \\
\hline Escamas laminares & $\begin{array}{l}\text { abaxial: muy esparcidas, } \\
\text { orbiculares, fimbriadas. } \\
\text { adaxial: muy esparcidas, orbicu- } \\
\text { lares y linear-lanceoladas }\end{array}$ & $\begin{array}{l}\text { abaxial: densamente esparcidas, } \\
\text { mixtas, de orbiculares a ovado- } \\
\text { apiculadas y caudadas, margen } \\
\text { fimbriado-ciliado. } \\
\text { adaxial: glabrescente }\end{array}$ & $\begin{array}{l}\text { abaxial: esparcidas, redondeadas a } \\
\text { aciculares, serruladas } \\
\text { adaxial: glabrescente }\end{array}$ \\
\hline Paráfisis & peltados, orbiculares & ovado-lanceolados a peltados & ausentes \\
\hline Esporas & monoletes, granulares a psiladas & $\begin{array}{l}\text { monoletes, abortivas, con grandes } \\
\text { verrugas }\end{array}$ & monoletes, con grandes verrugas \\
\hline
\end{tabular}

Akin to Pleopeltis $\times$ bartlettii (Weath.) A.R.Sm. $\mathrm{y}$ Tejero from which it differs by having a single type of scales on the rhizome, petiole shorter than the blade length and with scattered scales, as well as narrower blades, with scales on the abaxial surface mostly ovate and short-awned

\section{Descripción: in Farfán-Roldán et al. (2006).}

Distribución y hábitat: Planta epífita hasta ahora solo conocida de la localidad tipo en el Parque Nacional El Chico, al norte de la ciudad de Pachuca, estado de Hidalgo, en bosque mixto de pino y encino a $2880 \mathrm{~m}$ s.n.m.

Etimología: En honor a la botánica pteridóloga María de la Luz Arreguín Sánchez quien lleva una vida dedicada a la docencia y estudio de los helechos de México.

Comentarios: Farfán-Roldán et al. (2006) postulan mediante estudio comparativo morfológico y anatómico que las especies parentales son Pleopeltis polylepis var. polylepis y P. guttata (Maxon) E.G. Andreux \& Windham. Con la primera especie comparte el peciolo esparcidamente escamoso y la forma y coloración de dichas escamas y las del haz de la lámina. Con $P$. guttata comparte la ausencia de comas en las escamas del rizoma, la presencia de hidátodos en la lámina y la forma y coloración de las escamas del envés de la lámina. El nuevo híbrido y Pleopeltis $\times$ barlettii son similares, pero se pueden separar por los caracteres mencionados en la diagnosis y en la clave. Ambos híbridos se encuentran separados por una distancia geográfica notable (Hidalgo vs. Tamaulipas, $400 \mathrm{~km}$ en línea recta) y, dado la característica de sus esporas abortadas, es difícil hipotetizar dispersión a larga distancia. Por lo anterior, se consideran como dos híbridos diferentes.

\section{DISCUSIÓN}

El descubrimiento de un híbrido en Pleopeltis se basa a primera vista en la presencia de hojas anómalas (creci- 
miento o lobulación irregular). La existencia de esporas abortadas al menos en una proporción de más de $75 \%$ permite verificar el origen híbrido (Wagner et al., 1986). Generalmente las colonias de híbridos de Pleopeltis muestran caracteres con una clina de variación notable (sobre todo en la forma y disección de la hoja y tipo de escamas) entre las dos especies parentales que se correlaciona con el polimorfismo alélico (Hooper y Haufler, 1997). Sin embargo, en ocasiones alguno de los caracteres aparece constante y ello se puede corroborar con la observación en otras poblaciones cuando éstas se descubren. Considerando que en Polypodiaceae el fenómeno de hibridación es muy dinámico y forma parte de sus procesos de especiación/adaptación al epifitismo (Wagner y Wagner, 1975; Hooper y Haufler 1997; Haufler et al., 2000) y que México y Centroamérica son el centro de origen de cuatro subgrupos de Pleopeltis (Otto et al., 2009; Smith y Tejero-Díez, 2014), no es sorprendente que ahora se puedan reconocer en esta última región un total de 10 híbridos. Los híbridos más fáciles de distinguir en este género se dan entre grupos intragenéricos, con caracteres morfológicos extremos, como los del grupo de $P$. macrocarpa (Bory ex Willd.) Kaulf. de hoja simple y cualquiera de los otros de hoja pinnatífida o pinnadas reconocidos por Smith y Tejero-Díez (2014). Si se considera que en México las especies de Pleopeltis parentales más frecuentes de los híbridos encontrados (Pleopeltis fallax, P. guttata, P. madrensis, P. mexicana, P. plebeia, P. polylepis, P. polypodioides y $P$. thyssanolepis) son de amplia distribución y muy abundantes en las localidades de bosque templado y hábitats limítrofes y que la hibridación es un fenómeno natural en el ambiente de los helechos epífitos (Hooper y Haufler, 1997), se esperaría que aparecieran híbridos espontáneos repetidos, o nuevos, en más de una ocasión donde ocurriese coincidencia de las especies. El caso de Pleopeltis $\times$ arreguiniae/P. $\times$ barlettii, el descubrimiento en Puebla de varias poblaciones de $P$. ×melanoneuron previamente reportado para Veracruz y la presencia de $P$. $\times$ gracilis en un microambiente secundario densamente poblado por las especies parentales podrían ser claros ejemplos de ello. Farfán-Roldán et al. (2013) calculan la probabilidad y los sitios donde se podrían encontrar nuevas poblaciones de Pleopeltis $\times$ arreguiniae de acuerdo a la simpatría de las especies que ellos consideran implicadas. La variación morfológica de estos taxones híbridos ha sido muy poco estudiada debido a que en la mayoría de los casos solo se conoce la colonia descubierta y generalmente no hay más material disponible en los herbarios. Excepcionalmente se han recolectado, recientemente, varias colonias de Pleopeltis $\times$ melanoneuron, en cafetales abandonados existentes en buena parte de la zona montañosa en Cuetzalán, Puebla (E. Schuettpelz et al., 1787 y 1797; F!, MEXU!) que demuestran por un lado la posibilidad real de que el fenómeno de hibridación con las mismas especies progenitoras se repita y por otra parte, da la oportunidad de analizar algunos de los caracteres morfológicos y su variación, para así poder dilucidar cuales son dominantes paternales y cuales son intermedios. Del análisis de los híbridos conocidos y sus poblaciones se puede rescatar las siguientes tendencias.

1) Con excepción de Pleopeltis $\times$ gracilis, todos los nothotaxa están constituidos por una especie parental del grupo de $P$. macrocarpa, donde $P$. mexicana y $P$. polylepis son las mejor representadas. No es claro la dominancia de sus caracteres cuando forman híbridos; la forma de la lámina puede ser ahusada $(P . \times$ melanoneuron, $P . \times$ pueblensis y $P$. $\times$ sordidula) u ovado-deltada en el resto de los nothotaxa. En el primer caso llama la atención que la lámina solo se presenta con margen ondulado a lobado no importando el nivel de disección de su contraparte (pinnada en Pleopeltis fallax a pinnatífida en $P$. madrensis), pero en el segundo caso la lámina siempre estará de lobada a pinnatífida.

2) Pleopeltis fallax aparece como la especie más promiscua en México, no solo por la cantidad de especies con la que se relaciona, sino porque hibridiza con especies que pertenecen a dos subgrupos taxonómicos muy distintos en su morfología foliar (Smith y Tejero-Díez, 2014): P. macrocarpa y $P$. furfuracea. Lo interesante de estas relaciones es 
que en los híbridos resultantes domina el tipo de rizoma incluidas las escamas de Pleopeltis fallax (rizoma muy delgado, con escamas ovadas, diminutas, concolora o con un estrecho margen, producto de la contraparte parental, y comosas).

3) Considerando los híbridos de América, Pleopeltis thyssanolepis aparece como la especie parental con mayor difusión ya que ha producido los siguiente nothotaxa: Pleopeltis $\times$ aspidiolepis (Baker) A.R. Sm. (Costa Rica), P. ×leucospora (Klotzsch) T. Moore (Centro y Suramérica) y Pleopeltis $\times$ tricholepis en México. Esta especie además está en México muy relacionada con Pleopeltis fallacissima (Maxon) A.R. Sm. y Tejero y P. riograndensis (T. Wendt) E.G. Andrews y Windham del norte de México, especies que posiblemente son producto de hibridismo (Mickel y Beitel, 1987). Todos estos taxa muestran láminas ovadas, pinnatisectas con segmentos irregulares y con escamas en el peciolo.

4) El indumento escamoso en el rizoma y la hoja, aspecto que caracteriza a Pleopeltis (Otto et al., 2009), se hereda en los híbridos en forma mixta. Tanto la densidad, la forma y la combinación del tipo de celdillas que la constituyen, aparecen en los híbridos de dos maneras: con dominancia de alguno de los tipos escamoso parental (p. ej. P. × gracilis con dominancia de las escamas de $P$. villagranii en la lámina) o combinado (p. ej. las escamas del rizoma en $P$. $\times m e-$ lanoneuron con un margen estrecho) o la presencia de escamas no densamente dispuestas en el peciolo de los híbridos con P. thyssanolepis (p. ej. P. × tricholepis). Igual sucede con las comas de las escamas del rizoma; estas son generalmente características de algunas especies de Pleopeltis sensu stricto como $P$. astrolepis, $P$. fallax y $P$. mexicana. Los híbridos que conllevan a estas especies presentan una abundancia y distribución intermedia de las comas en las escamas del rizoma, tal como $P$. $\times$ sordidula. Es quizá el estado de variación de las escamas el principal argumento morfológico para establecer la paternidad de los híbridos en cuestión.
Con estas observaciones, se diseñó la siguiente clave dicotómica que permita a los usuarios una rápida determinación de los taxa híbridos mexicanos de Pleopeltis hasta ahora descubiertos (Fig. 3).

1a. Lámina ovada a deltoide; peciolo ca. 1/2 del tamaño de la hoja 2

1b. Lámina lineal a lanceolada; peciolo ca. 1/4 o menos del tamaño de la hoja

2a. Escamas del rizoma ocasionalmente con largas comas; lámina pinnatífida con 3-4 segmentos, porción apical subconformada con el segmento casi de la $1 / 2$ de la lámina P. $\times$ tricholepis

2b. Escamas del rizoma no comosas; lámina lobada a pinnatífida con 5 o más segmentos, porción apical graduada o cortamente subconformada con el segmento menor a $1 / 4$ de la lámina ................................ 3

3a. Escamas del rizoma mixtas; ovado-acuminadas y peltado caudado-lineares; peciolo densamente cubierto de escamas (ca. $75 \%$ cobertura); escamas abaxiales de la lámina mixtas, orbiculares u ovado-acuminadas P. $\times$ barlettii

3b. Escamas del rizoma monomorfas, ovado acuminadas a caudadas; peciolo espaciadamente escamoso $(<50 \%)$; escamas abaxiales de la lámina con dominancia de escamas ovado acuminada a aristadas

P. ×arreguiniae

4a. Rizoma esbelto (mayor a $1 \mathrm{~mm}$ de diámetro) con escamas (ápice meristemático) estrechamente ovado-acuminadas, $1.0-1.5 \times 0.4-0.6 \mathrm{~mm}$, fuertemente bicoloras, con una banda de celdillas clatradas a subclatradas negra y un ancho margen café claro

\section{P. ×pueblensis}

4b. Rizoma delgado (0.4-0.8 mm de diámetro) con escamas orbiculares a ovadas, menores a $1 \mathrm{~mm}$, concoloras o estrechamente marginadas 5

5a. Hojas manifiesta y regularmente pinnadas, gradualmente reducidas hacia el ápice; escamas de la lámina ovado-aristadas ................................... P. × gracilis

5 b. Hojas simples, irregularmente lobadas a pinnatisectas (si acaso con uno o dos segmentos libres en la 

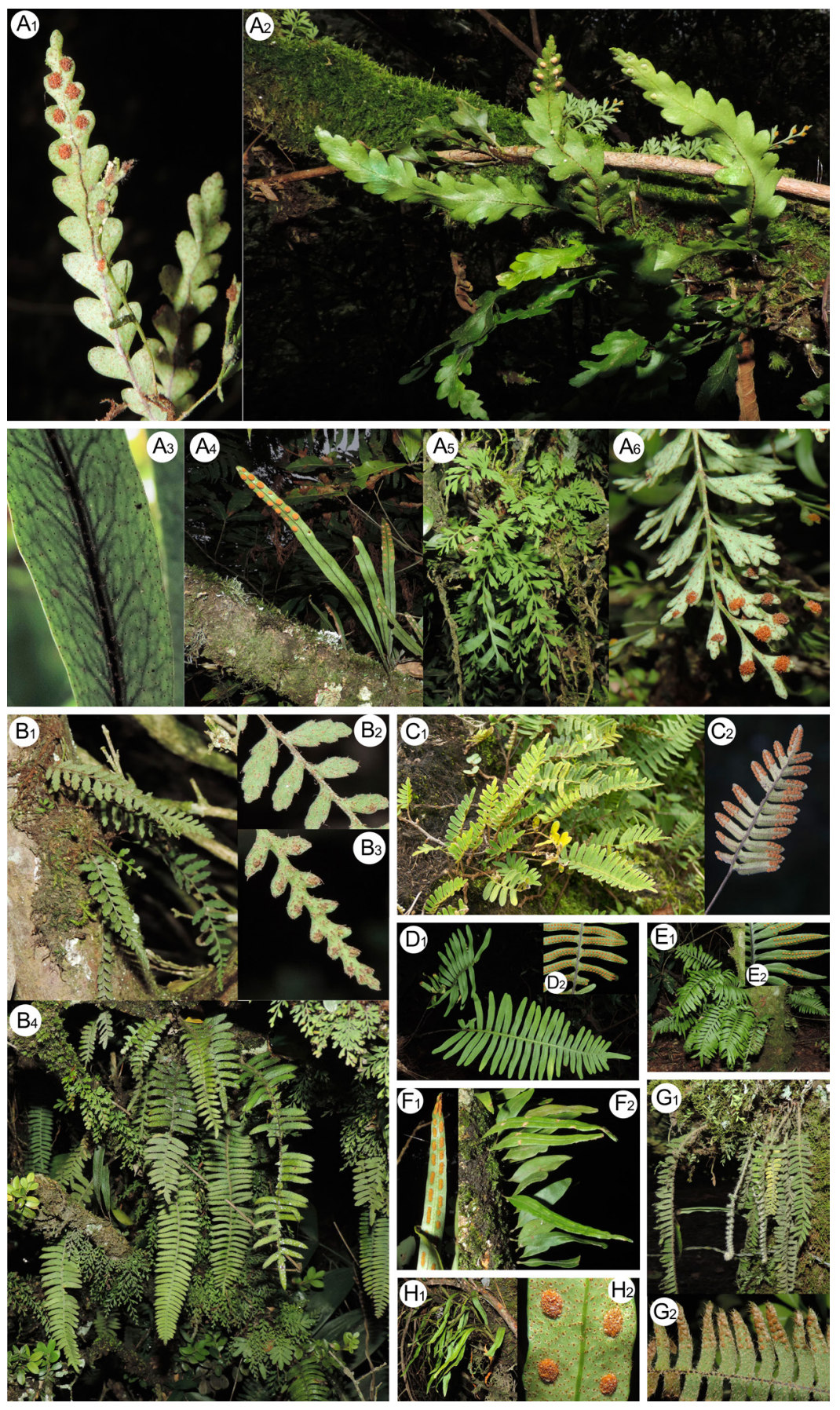

Figura 3: Lámina con fotografías de los híbridos y algunas de las especies parentales mencionadas en el trabajo. A. Pleopeltis $\times$ melanoneuron Mikel \& Beitel; $\mathrm{A}_{1}$. cara abaxial con soros; $\mathrm{A}_{2}$. hábito de la planta; $\mathrm{A}_{3}-\mathrm{A}_{6}$. especies parentales; $\mathrm{A}_{3}$ y $\mathrm{A}_{4}$. Pleopeltis crassinervata (Fée) T. Moore, mostrando detalles taxonómicos (venación a contraluz y hábito respectivamente); $\mathrm{A}_{5} \mathrm{y}_{6}$. P. fallax (Schltdl. \& Cham.) Mickel \& Beitel, hábito y detalles de la cara abaxial respectivamente. B. P. $\times$ gracilis A. Rojas \& Tejero; $\mathrm{B}_{1}-\mathrm{B}_{3}$. detalles de la planta, hábito y acercamientos en cara abaxial; $\mathrm{B}_{4}$. colonia mixta de P. villagranii (Copel.) A.R. Sm. \& Tejero y P. fallax bajo la protección de arbustos de Boj. C-H. especies de Pleopeltis Humb. \& Bonpl. ex Willd. que frecuentemente forman híbridos: C. P. polypodioides (L.) E.G. Andrews \& Windham; $\mathrm{C}_{1} \mathrm{y} \mathrm{C}_{2}$. hábito y cara abaxial mostrando soros. D. P. madrensis (J. Sm.) A.R. Sm. \& Tejero; $\mathrm{D}_{1}$ y $\mathrm{D}_{2}$. hábito y cara abaxial respectivamente. E. P. plebeia (Schltdl. \& Cham.) A.R. Sm. \& Tejero. E y E 2 . hábito y cara abaxial respectivamente. F. P. astrolepis (Liebm.) E. Fourn., $\mathrm{F}_{1}$ y $\mathrm{F}_{2}$. detalle de los soros en cara abaxial y hábito respectivamente. G. P. villagranii (Copel.) A.R. Sm. \& Tejero; $\mathrm{G}_{1} \mathrm{y} \mathrm{G}_{2}$. hábito y detalle de la cara abaxial mostrando soros y apariencia de las escamas. H. P. polylepis (A. Roem. ex Kunze) T. Moore var. polylepis; $\mathrm{H}_{1}$ y $\mathrm{H}_{2}$. hábito y detalles de la cara abaxial mostrando soros, escamas y costa verdosa. 
base); escamas de la lámina ovado-acuminados a mucronado ... 6

6a. Hoja con peciolo algo aplanado; lámina con las venas secundarias no visibles, irregularmente ondulada a lobada; escamas del rizoma orbiculares a ovadas, 0.2-0.3 mm de longitud P. $\times$ sordidula

6b. Hoja con peciolo terete; lámina que deja ver las venas secundarias en la porción basal; irregularmente lobada a seccionada; escamas del rizoma lanceoladas, 0.5-0.8 $\mathrm{mm}$ de longitud ........ P. $\times$ melanoneuron

Finalmente, la presencia de híbridos no solo está relacionada con aspectos de la biodiversidad, sino que son una herramienta para entender diferentes procesos de especiación y sobre todo pueden auxiliar a comprender las relaciones taxonómicas entre grupos intragenéricos, debido a la facilitación diferencial en la compatibilidad genética (Wagner, 1963). Para el caso de Pleopeltis, un género rico en especies a partir de la nueva circunscripción formulada por Smith y Tejero (2014), aún están mal entendidos los subgrupos y por lo tanto no se han designado subgéneros. Justamente Pleopeltis fallax tiene una posición infragenérica incierta y en los grupos de $P$. polypodioides, $P$. furfuracea y $P$. thyssanolepis entre otros, las relaciones aún son muy obscuras (Smith y Tejero, 2014). Por ejemplo, es muy sugerente que los estudios moleculares de Otto et al. (2009) indiquen una cierta relación entre los últimos grupos mencionados y que a su vez, alguna de las especies componentes sean muy activas, en los procesos de hibridación.

\section{CONTRIBUCIONES DE AUTORES}

ABCC, AFRA, LCO descubrieron los híbridos en campo, realizaron las descripciones morfológicas y contribuyeron con las figuras respectivas. JDTD verificó la taxonomía de las nuevas entidades, escribió el manuscrito, diseño la clave y las figuras. Todos los autores contribuyeron con la revisión y aprobación del manuscrito final.

\section{FINANCIAMIENTO}

Este estudio fue apoyado en lo general como parte de los proyectos que los autores tienen en las instituciones don- de laboran. Especialmente AFRA agradece a la Dirección de Investigación de la Universidad Nacional de Costa Rica, quienes a través del Fondo de Apoyo para el Fortalecimiento de Alianzas Estratégicas para el Desarrollo de Proyectos Colaborativos Internacionales financiaron su estancia en México.

\section{AgRADECIMIENTOS}

Al Dr. Alan R. Smith de la universidad de California en Berkeley por la revisión crítica de fotografías del material analizado (Pleopeltis × pueblensis). A los Dr. Fernando Chiang Cabrera y A.R. Smith por emitir opiniones respecto a la aplicación pertinente de los artículos del Código Internacional de Nomenclatura Botánica (por sus siglas en inglés ICBN) a los híbridos aquí tratados. Al Centro Universitario de Vinculación y Transferencia de Tecnología (CUVyTT), Benemérita Universidad Autonóma de Puebla (BUAP) y Dalia Grego Valencia de la Unidad de Morfología y Función (UMF) de la Facultad de Estudios Superiores Iztacala (FESI) por el valioso apoyo para la obtención de las microfotografías. Desde luego, la lectura crítica emitida por los dictaminadores anónimos y el editor de la revista auxiliaron a elevar el nivel académico y la claridad de este trabajo; a ellos un especial reconocimiento por su acuciosa labor.

\section{LITERATURA CITADA}

Aguilar-Rodríguez, S. 1998. Técnicas de laboratorio para el estudio de las embriófitas. In: Tejero Díez, J. D. y M. P. Granillo Velázquez (eds.). Plantae. Introducción al estudio de las plantas con embrión. 2a ed. Universidad Nacional Autónoma de México, Campus Iztacala. Tlalnepantla, México. Pp. 247-285.

Anthony, N. C. y E. A. Schelpe. 1985. $\times$ Pleopodium-a putative intergeneric fern hybrid from Africa. Bothalia 15: 555559. DOI: http://dx.doi.org/10.2307/1547633

Arreguín-Sánchez, M. L., J. Huerta-Zavala y A. GutiérrezBecerril. 2004. Un nuevo registro de Pleopeltis ${ }^{\times}$sordidula (Maxon ex Weath. in Weath) Mickel et Beitel, un híbrido entre Pleopeltis astrolepis (Liebm.) Fourn. y Pleopeltis fallax (Schldl. et. Cham.) Mickel et Beitel 
(Polypodiaceae, Pteridophyta) en Tlaxcala, México. Polibotánica 17: 39-43.

Barrington, D. S., C. H. Haufler y C. R. Werth. 1989. Hybridization, reticulation, and species concept in ferns. American Fern Journal 79(2): 55-64. DOI: http://dx.doi. org/10.2307/1547160

Chang, H. M., W. L. Chiou y J. C. Wang. 2009. Molecular Evidence for Genetic Heterogeneity and the Hybrid Origin of Acrorumohra subreflexipinna from Taiwan. American Fern Journal 99(2): 61-77. DOI: http://dx.doi. org/10.1640/0002-8444-99.2.61

Farfán-Roldán, I., C. Gómez-Alanís y M. L. Arreguín-Sánchez. 2006. Un nuevo híbrido para México de Pleopeltis polylepis (Roemer ex Kunze) T. Moore var. polylepis $\times$ Polypodium guttatum Maxon. Polibotánica 22: 9-19.

Farfán-Roldán, I. M., Y. Salinas-Moreno, O. Mejía, S. Acosta-Castellanos y M. L. Arreguín-Sánchez. 2013. Distribución potencial del híbrido Pleopeltis polylepis (Roemer ex Kunze) T. Moore var. polylepis $\times$ Polypodium guttatum Maxon (Polypodiaceae-Pteridophyta): Áreas probables de simpatría. Polibotánica 36: 63-78.

Gómez-Pignataro, L. D. 1975. Contribuciones a la pteridología costarricense. VIII. La hibridación en el trópico: Microgramma $\times$ Polypodium y $P$. aspidiolepis Baker. Brenesia 6: 49-57.

Haufler, C. H., M. D. Windham y E. W. Rabe. 1995. Reticulate evolution in the Polypodium vulgare complex. Systematic Botany 20(2): 89-109. DOI: http://dx.doi. org/10.2307/2419442

Haufler, C. H., E. A. Hooper y J. P. Therrien. 2000. Models and mechanisms of speciation in pteridophytes: Implications of contrasting patterns in ferns representing temperate and tropical habitats. Plant Species Biology 15: 223-236.

Hooper, E. A. y C. H. Haufler. 1997. Genetic diversity and breeding system in a group of neotropical epiphytic ferns (Pleopeltis; Polypodiaceae). American Journal of Botany 84(12): 1664-1674. DOI: http://dx.doi. org/10.2307/2446464

JSTOR. 2000 a 2016. JSTOR Global Plants. Base de datos ITHACA. https://plants.jstor.org/ (consultado junio de 2015).
McNeill, J., F. R. Barrie, W. R. Buck, V. Demoulin, W. Greuter, D. L. Hawksworth, P. S. Herendeen, S. Knapp, K. Marhold, J. Prado, W. F. Prud'homme Van Reine, G. F. Smith, J. H. Wiersema y N. J. Turland. 2012. International Code of Nomenclature for algae, fungi and plants. (Melbourne Code). International Association for Plant Taxonomy. http: //www.iapt-taxon.org/nomen/main.php (consultado noviembre de 2014).

Mickel, J. T. y J. M. Beitel. 1987. Notes on $\times$ Pleopodium and Pleopeltis in Tropical America. American Fern Journal 77(1): 16-27. DOI: http://dx.doi.org/10.2307/1547633

Mickel, J. T. y A. R. Smith. 2004. The Pteridophytes of Mexico. Memories of the New York Botanical Garden 88: 1-1054.

Otto, E. M., T. Janssen, H. P. Kreier y H. Schneider. 2009. New insights into the phylogeny of Pleopeltis and related neotropical genera (Polypodiaceae, Polypodiopsida). Molecular Phylogenetics and Evolution 53(1): 190-201. DOI: http://dx.doi.org/10.1016/j.ympev.2009.05.001

Reyes-Carrada, A. 2012. Un probable nuevo híbrido de Pleopeltis $\times$ Polypodium del estado de Michoacán, México. Tesis de licenciatura. Instituto Politécnico Nacional, Escuela Nacional de Ciencias Biológicas. México D.F., México. $59 \mathrm{pp}$.

Sigel, E. M., M. D. Windham, C. H. Haufler y K. M. Pryer. 2014. Phylogeny, divergence time estimates, and phylogeography of the diploid species of the Polypodium vulgare complex (Polypodiaceae). Systematic Botany 39(4): 1042-1055. DOI: http://dx.doi. org/10.1600/036364414X683921

Smith, A. R. y J. D. Tejero-Díez. 2014. Pleopeltis (Polypodiaceae), a redefinition of the genus and nomenclatural novelties. Botanical Sciences 92(1): 43-58.

Wagner, Jr., W. H. 1969. The role and taxonomic treatment of hybrids. BioScience 19(9): 785-789+795. DOI: http://dx. doi.org/10.2307/1294787

Wagner, W. H. Jr. y F. S. Wagner 1975. A hybrid polypody from the New World tropics. The Fern Gazette 11: 125-135.

Wagner, Jr., W. H. F. S. Wagner y W. C. Taylor. 1986. Detecting Abortive Spores in Herbarium Specimens of Sterile Hybrids. American Fern Journal 76(3): 129-140. DOI: http:// dx.doi.org/10.2307/1547721 